\title{
The role of self-care and the use of smart sensors in the UK's health provision
}

Abstract. Self-care is an inseparable part of maintaining health, recovering from acute illnesses and living with long-term conditions. The individual's health literacy, personal goals, circumstances and opportunities all play a part. Person-centred healthcare recognizes this and seeks to work with the patient to help them achieve the best outcomes. This paper explores the evidence regarding how twoway electronic data flows between patients and their general practice may contribute to self-care, and explores new systems being developed to facilitate such flows.

Nanotechnology Perceptions 13 (2017) 5-16

doi: 10.4024/N15SU16A.ntp.13.01 